\title{
Citrobacter rodentium, a Gut Pathogen: The Yin and the Yang of Its Pathophysiology, Immunity and Clinical Manifestation in Mice
}

\author{
Tania Rahman ${ }^{1,2 *}$, Md. Ferdous Seraj ${ }^{3}$, Md. Monirul Islam ${ }^{4}$ \\ ${ }^{1}$ Department of Biochemistry and Molecular Biology, Bio21 Molecular Science and Biotechnology Institute, University of \\ Melbourne, Melbourne, Australia \\ ${ }^{2}$ Department of Microbiology and Immunology, University of Melbourne at the Peter Doherty Institute for Infection and \\ Immunity, Melbourne, Australia \\ ${ }^{3}$ School of Civil, Environmental and Chemical Engineering, RMIT University, Melbourne, Australia \\ ${ }^{4}$ Fiona Elsey Cancer Research Institute, Ballarat Technology Park Central, Ballarat, Australia \\ Email: *tania.rahman@du.ac.bd
}

How to cite this paper: Rahman, T., Seraj, Md.F. and Islam, Md.M. (2018) Citrobacter rodentium, a Gut Pathogen: The Yin and the Yang of Its Pathophysiology, Immunity and Clinical Manifestation in Mice. Advances in Microbiology, 8, 699-718.

https://doi.org/10.4236/aim.2018.89047

Received: June 15, 2018

Accepted: August 20, 2018

Published: September 17, 2018

Copyright (C) 2018 by authors and Scientific Research Publishing Inc. This work is licensed under the Creative Commons Attribution International License (CC BY 4.0).

http://creativecommons.org/licenses/by/4.0/

\begin{abstract}
Pathogenic strains of E. coli including enteropathogenic E. coli (EPEC), enterohemorrhagic E. coli (EHEC), enterotoxigenic E. coli (ETEC) are principle causes for diarrhoea in many parts of the globe. Citrobacter rodentium ( $C$. rodentium), a gram negative bacterium, is a murine pathogen that also utilizes type III secretion system and similar virulence factors to EPEC and EHEC and forms comparable attaching/effacing lesions in the intestines as EPEC and EHEC. The infection caused by $C$. rodentium in mice is usually self-limiting and results in only minor systemic effects with higher mortality in some susceptible mouse strains. All these characteristics have made the bacteria a commonly used model to study host immune responses to pathogenic $E$. coli infection. In this review, we focus on the impact of virulence factors of the pathogen; different immune components involved in the immune response and summarize their role during $C$. rodentium infection.
\end{abstract}

\section{Keywords}

Citrobacter rodentium, Attaching and Effacing Pathogen, Locus of Enterocyte Effacement, Transmissible Murine Colonic Hyperplasia, Colitis, Mucosal Immune Response

\section{Introduction}

Escherichia coli is a frequent commensal organism of human intestine, often 
colonizing immediately after birth and usually remaining for decades [1]. However, some E. coli strains exhibit pathogenic potential, when they acquire certain virulence associated genes [2]. Pathogenic strains of $E$. coli including enteropathogenic E. coli (EPEC) and enterohemorrhagic E. coli (EHEC) are the leading causes of diarrheal outbreak in most parts of the world [3]. EPEC is a major source of diarrhoea in children under two years of age causing deaths of a million each year in developing countries [4] [5]. EHEC causes bloody diarrhoea in children and elderly in developed countries with approximately 73,000 cases reported each year in the United states [6] [7] which can cause fatal diseases like haemorrhagic colitis and haemolytic uremic syndrome [8] [9]. The hallmark of EPEC and EHEC induced pathology is that they populate in the intestinal epithelium through the development of attaching and effacing (A/E) lesions [10]. As these pathogens are a profound global health concern understanding their clinical manifestation, pathogenesis and immunity has become the focus of extensive investigation. However, EPEC and EHEC elicit narrow range of host specificity and do not elicit a pertinent disease in laboratory animal genera, which makes it difficult to study EPEC and EHEC pathogenesis [11]. Citrobacter rodentium is an accepted mouse pathogen that uses comparable virulence factors as EPEC and EHEC and forms analogous A/E lesions in the distal colon of mice [12] [13] [14]. Subsequently, C. rodentium has become a commonly used animal representative to explore the immune responses to pathogenic $E$. coli contamination in humans.

\section{2. $C$. rodentium, an Attaching and Effacing Pathogen}

C. rodentium (formerly known as Citrobacter freundii biotype 4280) a non-motile, gram-negative bacteria in the family of Enterobacteriaceae, is recognised as the contributing species of transmissible murine colonic hyperplasia (TMCH) [15]. The disease is spread through the faecal-oral route and clinical symptoms occur predominantly in weanling mice [16]. Overall, the infection is self-curing in adult mice with higher mortality in some susceptible mouse strains [13]. In general, the infected mice exhibit decrease in body weight, excretion of soft faecal pellets with diarrhoea in severe cases and crypt hyperplasia.

Following entry to the body via oral route, $C$. rodentium colonizes the caecal patch, a type of lymphoid tissue in caecum, from where the bacteria gradually progress to colonise distal colon [17] [18] [19]. For colonization, the bacteria triggers localised damage of brush border microvilli, which mediates bacteria to attach to the epithelial cell surface. This attachment subsequently produces actin-rich structures of epithelial cells which resemble pedestal and they form these lesions beneath the associated bacteria [20]. The ultra-structural alterations are known as attaching and effacing lesions [21] [22] [23]. The effector proteins essential for the generation of $\mathrm{A} / \mathrm{E}$ lesion are transported to an intestinal cell through a type III secretion system (Figure 1), a needle-like structure, which ensures a smooth passage of bacterial proteins directly in to the host cell cytoplasm. 


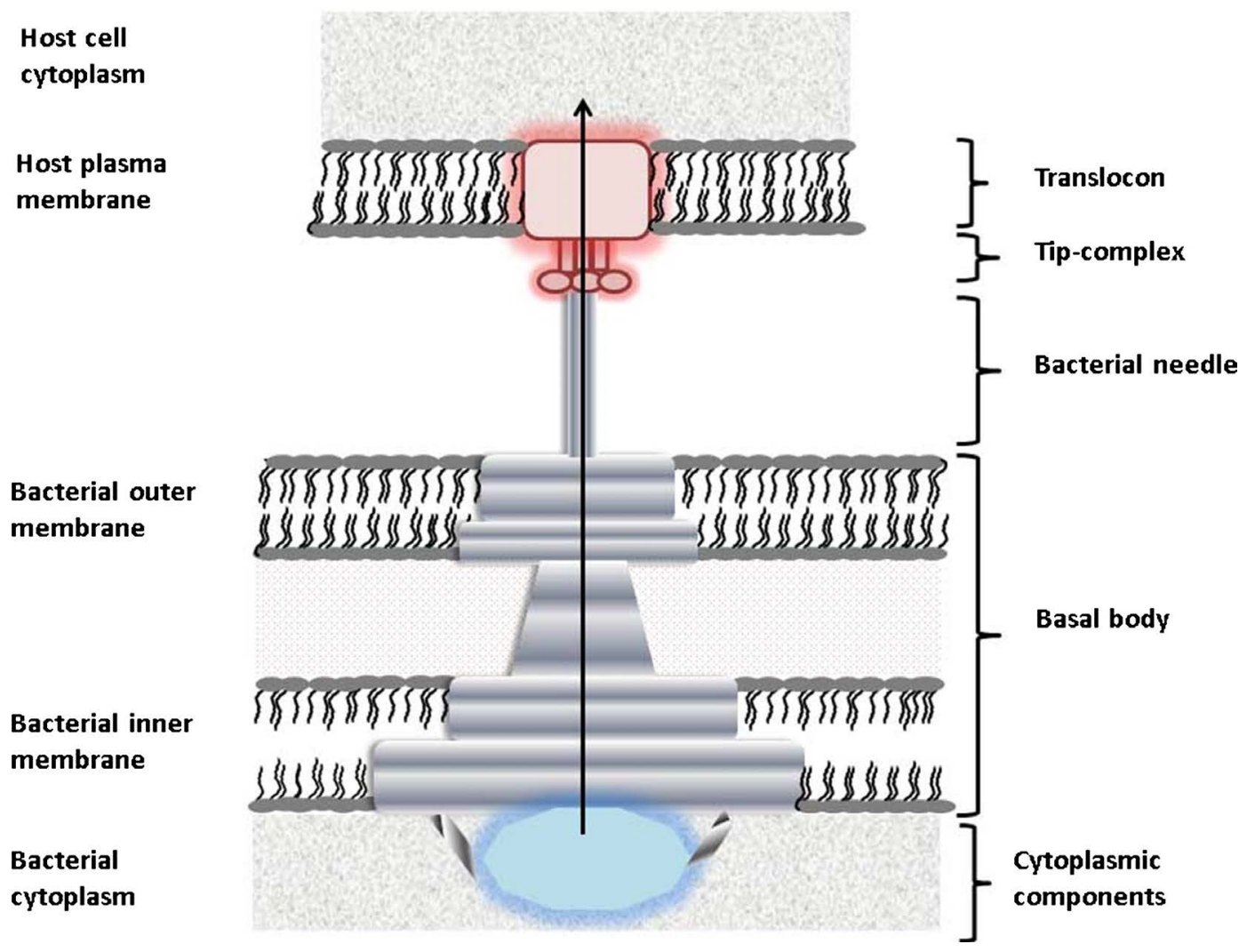

Figure 1. Illustration of type III secretion system (T3SS) in C. rodentium. The T3SS consists of a complex apparatus that actively delivers bacterial effector proteins into the cytoplasm of a host cell. The basal body spans the bacterial membranes and a needle-like syringe extends from the surface which is capped by a tip complex. Upon host cell contact, a translocon is inserted in the host cell membrane and forms a pore. Bacterial virulence proteins are then selectively secreted through the syringe into the host cell, where they manipulate host cell functions essential for subsequent pathogenicity.

\subsection{Virulence Factors of $C$. rodentium}

The type III secretion system is programmed by a cluster of genes recognized as locus of enterocyte effacement (LEE), a conserved pathogenicity island consisting of $35.6 \mathrm{~kb}$ [24]. The LEE pathogenicity island consists of over 40 genes which are organised in to five operons including LEE1, LEE2, LEE3, LEE4 and LEE5 [24] [25] [26]. LEE encodes several structural components of T3SS, effectors, translocators and several other proteins (Figure 2) [27] [28]. One important effector protein is Tir (translocated intimin receptor) a bacteria derived receptor, which following translocation binds to the host epithelial cell and interacts with intimin, the bacterial outer membrane protein, thereby facilitates anchorage of bacteria to host cell, leading to pedestal formation [29] (Figure 3). The intimins are encoded by eae genes that are extremely conserved in $\mathrm{N}$-terminal regions, however, display substantial heterogeneity at the C-termini [30]. Five different intimin types $\alpha, \beta, \gamma, \delta$ and $\varepsilon$ have been recognized [31] [32]. Intimin $\alpha$ and intimin $\beta$ are expressed mainly by strains pertaining to EPEC clones 1 and 2, correspondingly, whereas intimin $\gamma$ is expressed by enterohaemorrhagic E. coli (EHEC) serotype O157:H7 and intimin $\delta$ by EPEC O86:H34 [33]. 


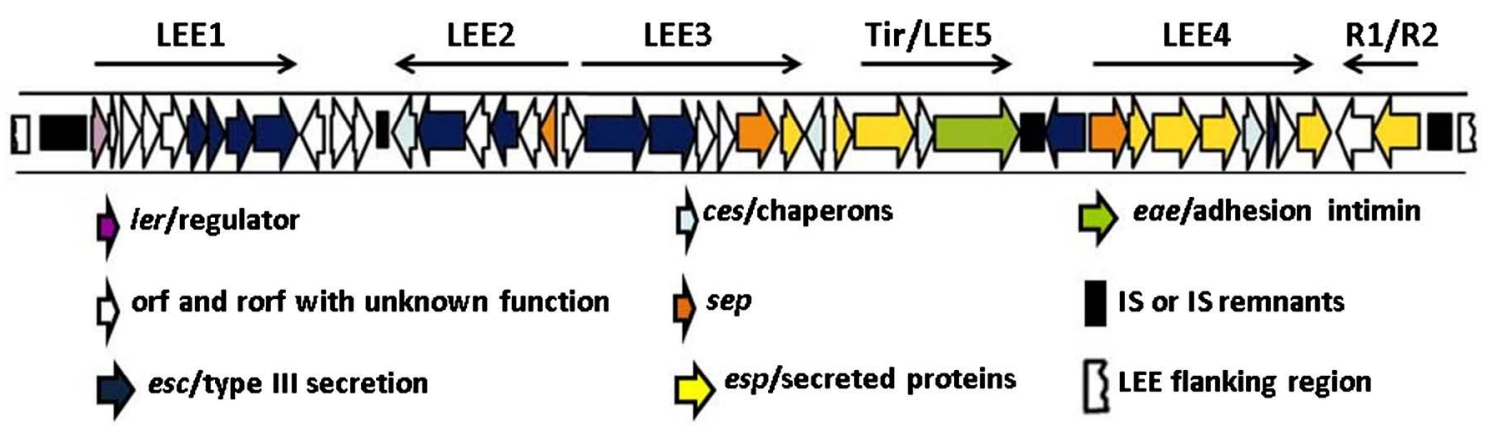

Figure 2. Genetic assembly of $C$. rodentium LEE. The orientation of each gene is shown by the direction of the arrow. The different locations of the rorfl $(\mathrm{r} 1)$ and $\operatorname{rorf2}(\operatorname{esp} G / \mathrm{r} 2)$ genes in $C$. rodentium LEE, as well as the association of several IS's or IS remnants with the $C$. rodentium LEE. The major operons encoded by the LEE (LEE1, $-2,-3$, and -4 , Tir, and R1/R2) and their transcriptional directions are shown and adapted from reference [25].

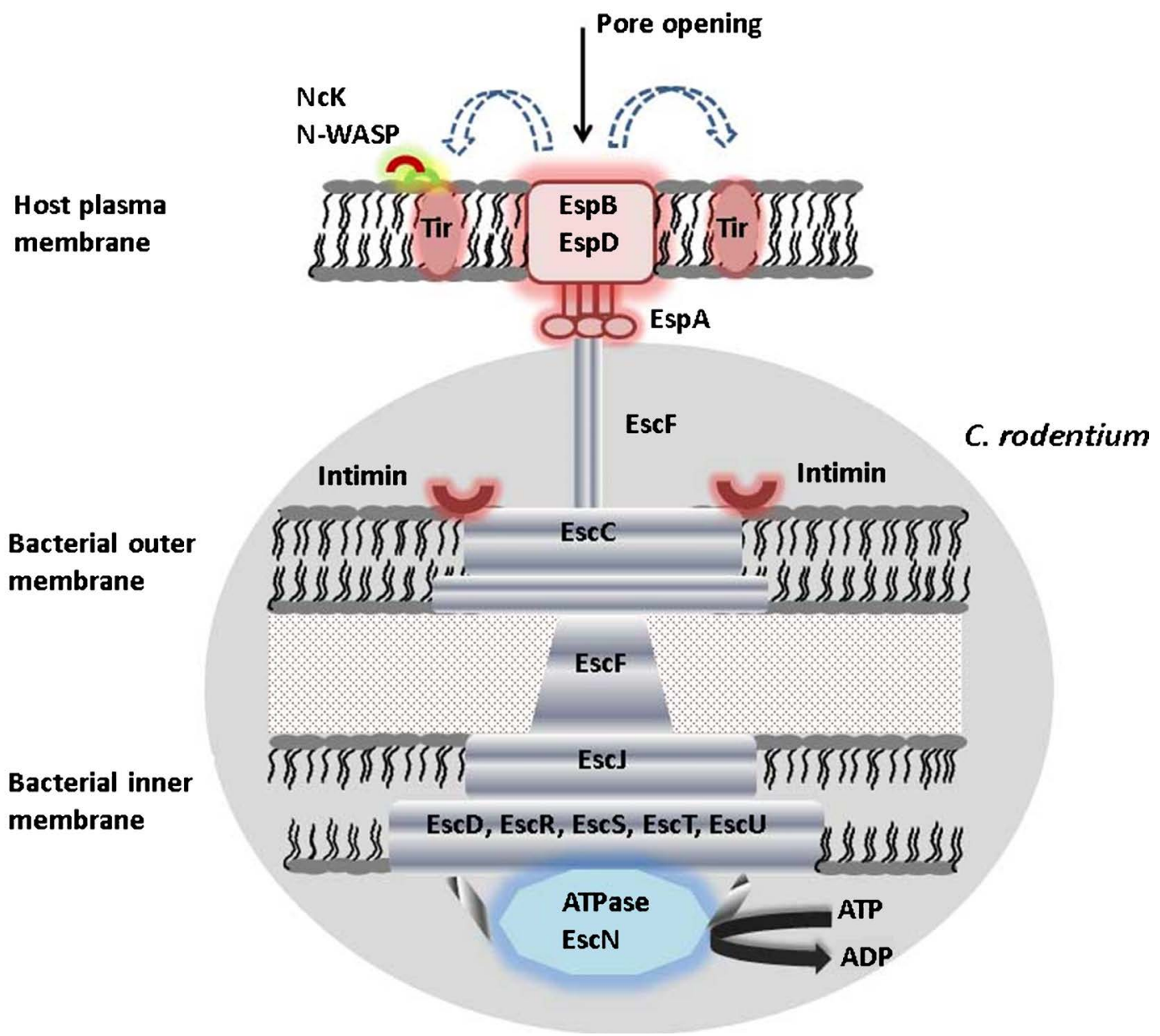

Figure 3. Translocation of $C$. rodentium secreted proteins occurs through a LEE-encoded type III secretion system that spans the inner and outer membranes of the bacteria as well as that of the host cell. EscJ is the inner membrane ring which forms the platform for all other Type III secretion components. EspA-containing surface organelles form a filamentous tube for the translocation into the host cell cytosol. EspB and EspD are then inserted into the host cell membrane, form a pore structure in the plasma membrane enabling the passage of bacterial effectors, such as Tir. Tir serves as a receptor for the outer membrane ligand, intimin. Tir and intimin interaction initiates a signal transduction cascade that aids in the recruitment of actin to form attaching and effacing (A/E) pedestals beneath the adherent bacteria. Tir molecules recruit Nck leading to the activation of N-WASP and strong actin polymerization. 
Upon entry to the host cells, Tir is tyrosine phosphorylated, which recruits non-catalytic region of tyrosine kinase adaptor protein Nck [34] [35] [36] [37]. Nck binds to the phosphorylated tyrosine and this in turn triggers the recruitment of nucleation promoting factor N-WASP (neural Wiscott-Aldrich syndrome protein) and actin-regulated protein Arp2/3 complex, resulting in host actin rearrangement [38] [39] [40]. Tir triggers localized actin polymerization by another two different pathways. After translocation, LEE and non-LEE effectors contribute to the impediment of several signalling pathways in the host cell, including actin polymerization, tight junction integrity, endosomal trafficking, apoptosis, phagocytosis and innate immune responses, as well as epithelial cell shedding and detachment [41].

Besides Tir, LEE encodes several secreted translocators: EspA, EspD, EspB, EspF, EspG, EspH, EspZ, which are entirely translocated into the host cells and are involved in modulating host cytoskeleton leading to the manifestation of disease [4] [42] [43]. A/E pathogens secrete numerous LEE-encoded regulatory proteins, Ler, GlrA and GlrR, which exhibit a significant role in the transcriptional regulation of LEE and several non-LEE virulence determinants [44] [45] [46]. Moreover, RegA adjusts LEE expressions through upregulating $g r l R / A$ transcription [47]. There are several effectors that are not secreted and translocated by the LEE-encoded T3SS including prophages and insertion sequences. They comprise the Espl/NleA, an indispensable protein for entire virulence of $C$. rodentium [48] [49] [50] and binds host PDZ-domain proteins [51]; EspB, essential for intimate attachment and signal transduction (Figure 3) [52]; EspJ, which display a negligible part in enteric colonization [53]; and EspG, stimulates the dissociation of microtubules beneath adherent bacteria [54]. In addition, two non-LEE encoded proteins, NleB to NleH, are found in C. rodentium and they are mostly produced by the LEE-encoded T3SS [49] [55]. Among these, NleC and NleD have been recognized to be translocated into host cells [56]. NleB1 binds to host cell death domain encoding proteins, diminishes the signalling of a death receptor and thereby disrupting a major antimicrobial host response [57].

Other than the genes for rorfl and rorf2/espG and several insertion sequences (IS) and IS remnants, both the LEE of $C$. rodentium and that of EPEC and EHEC shares all 41 ORFs and the linear gene sequences (Figure 2) [25]. This suggests that the LEE encoded pathogens has a mutual evolutionary origin and reciprocal function which supports the use of $C$. rodentium as an animal model to study A/E pathogenesis.

\subsection{Disease Progression of $C$. rodentium}

Similar to EPEC and EHEC, C. rodentium infection encompasses three distinct phases: 1) an initial colonization phase specially facilitated by bacterial effector proteins, 2) an acute phase characterized by colonic hyperplasia with the initiation of diarrhoea in severe cases, 3) a convalescent phase manifest as the clearance of bacteria and the prevention of further invasion. 
During the first week after inoculation, $C$. rodentium colonizes the brush border microvilli and higher numbers of bacteria are seen closely adherent to the mucosal epithelial cells (Figure 4) [59] [60]. Acute phase of infection follows over the subsequent 2 weeks when the levels of bacteria peak around $>10^{9}$ c.f.u per gram of tissue in the colon [16] and the bacteria induce a profound hyperplasia of colonic mucosa with the development of secretory diarrhoea [61]. At the time of peak hyperplasic phase, the organism can no longer be isolated from the intestines and the infected mucosa are thickened markedly. Convalescent phase of infection comes following 4 weeks and above, for the period of which the reactive epithelial hyperplasia to clinical diarrhoea get resolved and colonic mucosa appears normal [13] [62].

\subsection{Immune Defense against $C$. rodentium}

Most of the existing knowledge on the immune response and its relation with pathology has been expanded using mice with the targeted ablations of various immune components. Innate immune response as well as adaptive immune response appears to control mucosal defence against $C$. rodentium [63].

\subsubsection{Role of Adaptive Mucosal Immune Responses}

The concept of exploring mice with deficiencies in immune components first came from the study that colonic mucosa of infected mice contained large infiltrates of $\mathrm{CD}^{+} \mathrm{T}$ cells with a helper $\mathrm{T}$ cell 1 cytokine response [64]. Substantial mortality was observed in mice deficient in $\mathrm{CD}^{+}{ }^{+} \mathrm{T}$ cells or TCR $\alpha \beta+\mathrm{T}$ cells [59]. Mice lacking CD4 showed a survival limit of two weeks and exhibited $100 \%$

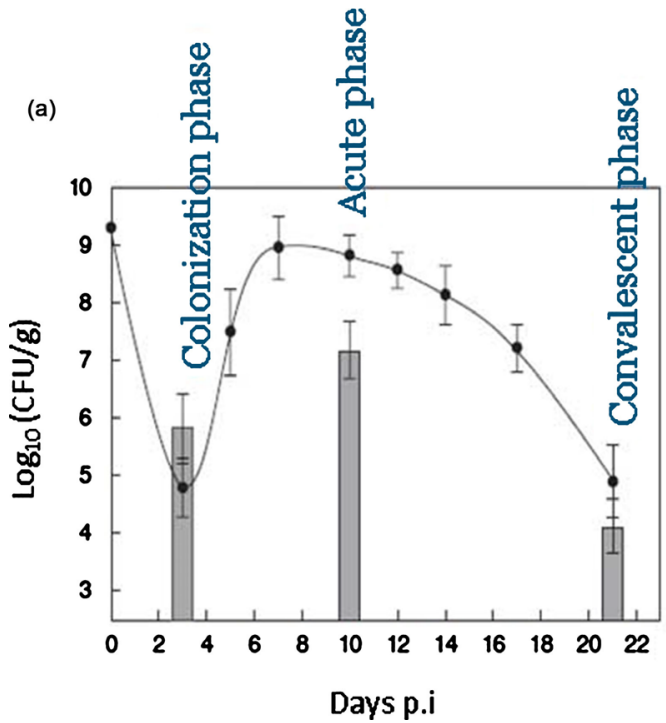

(b)

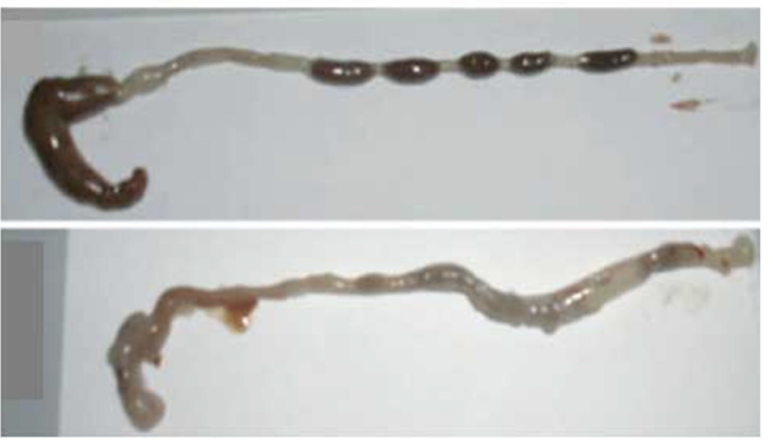

Figure 4. Infection kinetics in $\mathrm{C} 57 \mathrm{BL} / 6$ mice infected with $C$. rodentium. (a) C. rodentium bacterial burden at three distinct phases of infection: A colonization phase which persists from day 3 to day 5 after infection, an acute phase manifest as diarrhoea and host inflammatory responses and a convalescent phase during which the host clears pathogen (partially adapted from [58]); (b) The image here represents the gastrointestinal tract isolated from a normal (upper one) and an infected mouse (lower one). Infection results in empty and retracted caecum and a thickened colon devoid of normal stool (partially adapted from [12]). 
mortality. However, depletion of $\mathrm{CD}^{+} \mathrm{T}$ cells or $\mathrm{TCR} \gamma \delta^{+} \mathrm{T}$ cells did not adversely affect survival of infection and played a minor role in surviving the acute phase of infection. Two studies [63] [65] [66] separately demonstrated the vital significance of B cells for the protective immunity against $C$. rodentium. Mice lacking mature B cells ( $\mu \mathrm{MT}$ mice) failed to mount early inflammatory response at 2 weeks and could not lessen bacterial load or clear bacterial colonization over a prolonged period [63] [65] [66]. RAG1-deficient mice in which both B and T cells were absent displayed chronic intestinal colonization and more severe colonic damage and these mice were unable to clear infection and died after 3-4 weeks [59] [63] [67].

To analyse the potential of secretory antibodies in bacterial clearance, mice with selective deficiencies for IgA, IgM or IgG were used [65]. Both IgA- and IgM- deficient mice had been found to develop effective immunity against a secondary challenge and played a negligible role in controlling $C$. rodentium infection. However, mice deficient in IgG antibodies lost the ability to develop robust protective response against secondary challenge. Thus host defense against C. rodentium was dependent on IgG antibodies but did not require secretion of IgA or IgM [65]. A comparative analysis of the specific ablation of different adaptive immune components is summarized in Table 1.

Simmons and co-workers investigated $C$. rodentium infection in IFN $\gamma$-deficient and IL-12 deficient mice. IFN $\gamma$-deficient mice had higher bacterial numbers and

Table 1. Summary of the effects of selective ablation of adaptive immune components on C. rodentium infection in mice.

\begin{tabular}{|c|c|c|}
\hline Mouse models & Effects of ablation of specific adaptive immune components on $C$. rodentium infection & Refs \\
\hline $\begin{array}{l}\text { Mice lacking } \mathrm{CD}^{+} \mathrm{T} \text { cells or } \\
\mathrm{TCR} \alpha \beta+\mathrm{T} \text { cells }\end{array}$ & A survival limit of two weeks and exhibited $100 \%$ mortality & [59] [64] \\
\hline $\begin{array}{l}\text { Mice lacking } \mathrm{CD}^{+} \mathrm{T} \text { cells or } \\
\mathrm{TCR} \gamma \delta^{+} \mathrm{T} \text { cells }\end{array}$ & Do not adversely affect survival of infection & [59] \\
\hline $\begin{array}{l}\text { Mice without mature B cells } \\
\text { ( } \mu \mathrm{MT} \text { mice) }\end{array}$ & Cannot lessen bacterial load or clear bacterial colonization over a prolonged period & $\begin{array}{l}{[63][65]} \\
{[66]}\end{array}$ \\
\hline $\begin{array}{l}\text { RAG1-deficient mice (both B } \\
\text { and T cells are absent) }\end{array}$ & Develop chronic intestinal colonization and unable to clear infection and die after 3-4 weeks & $\begin{array}{l}{[59][63]} \\
{[67]}\end{array}$ \\
\hline IgA- and IgM- deficient mice & $\begin{array}{l}\text { Develop effective immunity against a secondary challenge and play a negligible role in controlling } C \text {. } \\
\text { rodentium infection. }\end{array}$ & {$[65]$} \\
\hline IFN $\gamma$-deficient mice & $\begin{array}{l}\text { Higher bacterial numbers and enhanced mucosal thickening in colons and cannot clear infection until } \\
\text { day } 28\end{array}$ & {$[68]$} \\
\hline IL-12 deficient mice & $\begin{array}{l}\text { Elicit higher bacterial numbers for the first } 3 \text { weeks of infection and eventually clear infection by day } \\
35\end{array}$ & {$[30]$} \\
\hline Mice lacking IL-22 & $\begin{array}{l}\text { Display systemic bacterial load and enhanced epithelial hyperplasia and mortality range up to } 100 \% \\
\text { within the first two weeks of infection }\end{array}$ & {$[69]$} \\
\hline $\begin{array}{l}\text { Treg deficient mice (DEREG } \\
\text { mice) }\end{array}$ & $\begin{array}{l}\text { Diminished bacterial clearance, systemic dissemination of bacteria with compromised Th17 immune } \\
\text { response accompanied by less inflammation-associated pathology }\end{array}$ & {$[70]$} \\
\hline IL-10 ablated mice & Resolve infection earlier than wild-type mice with less infection associated colitis & [71] \\
\hline
\end{tabular}


enhanced mucosal thickening in their colons and could not clear infection until day 28 [68]. Alternatively, IL-12 deficient mice had been shown to elicit higher bacterial numbers for the first 3 weeks of infection and eventually cleared infection by day 35 [30]. Interleukin-22 (IL-22) has been identified as an essential cytokine for mediating protection against $C$. rodentium infection. While compared to wild-type mice, mice lacking IL-22 infected with $C$. rodentium displayed systemic bacterial load and enhanced epithelial hyperplasia. In multiple repeat experiments, the mortality of IL-22 knockout mice ranged from $80 \%$ to $100 \%$ within the first two weeks after infection [69]. Administration of Reg3 $\gamma$, an antimicrobial peptide to IL-22 knockout mice controlled infection.

During infection, $\mathrm{CD}^{+}$Th17 cell subsets were particularly amplified in Peyer's patches (PP) but were unaltered in mesenteric LNs [70]. The differentiation of Th17 cells in PP were dependent on the inflammatory cytokine IL-6 as treatment with anti-IL- 6 antibodies reduced Th17 cells and exacerbated clinical manifestation of colitis. Moreover, following anti-IL-6 antibody treatment, there was a reduction of IL-22 mRNA expression in the small intestine during infection but had no effect on IgA production by B cells [70].

Symonds and co-workers demonstrated an elevated FoxP3 mRNA expression in the distal colon at all stages of infection with C. rodentium. Also, C. rodentium infection exhibited an up-regulation of IL17 mRNA expression [58]. Wang and co-workers investigated role of Treg during $C$. rodentium infection using DEREG mouse model. Depletion of Treg by diphtheria toxin led to a diminished bacterial clearance and systemic dissemination of bacteria. Also, Th17-associated immune response was compromised following Treg-depletion, with less inflammation-associated pathology in the colons of Treg-depleted mice. Treatment with Anti-IL2 in depleted mice retained Th17 induction, suggesting that Treg induced a protective Th17 response by intake of local IL-2 [71]. IL-10 was found dispensable in controlling inflammation as IL-10 ablated mice resolved infection earlier than wild-type mice and had less infection associated colitis [72]. In addition, following infection, IL-27 was produced which subsequently suppressed Th17 in vitro and thus play role in anti-inflammatory circuit in the absence of IL-10. The neutralization of IL-27 led to pronounced colitis in mice lacking IL-10 suggesting that IL-10 enacts a minor part in the bacterial clearance whereas IL-27 might be an important cytokine for attenuation of inflammation [72].

Th22 cells were found to be important in the mucosal anti-microbial host defense against C. rodentium. Basu and co-workers [73] demonstrated that C. rodentium induced a wave of IL-22 producing ILCs and $\mathrm{CD} 4^{+} \mathrm{T}$ cells were each critical to host protection during infection. Though the IL-22 production by ILCs was strictly IL-23 dependent, IL-22 production by CD4 cells was not IL-23 dependent rather the production was dependent on IL- 6 and transcription factors T-bet and AhR. Also IL-22 producing CD4 cells (Th22) were more effective in host protection than Th17 cells. IL-17 was found to be important in host defense against $C$. rodentium [74]. The bacterial burden in the colon after infection 
with C. rodentium showed similar increases in IL-17f-/-, IL17a-/-, and IL-17a-/-IL17f-/- mice, indicating that deficiency of just one of the IL-17 proteins resulted in full susceptibility to infection. However, splenomegaly and colon hypertrophy, which were associated with severe colonic inflammation, were more pronounced in IL-17f-/- mice than in IL-17a-/- mice suggesting that IL-17f was more important than IL-17a in protecting colonic epithelial cells from the pathogenic effects of this bacterium.

\subsubsection{Role of Innate Mucosal Immune Responses}

There are several innate immune components which perform a vital role in mucosal homeostasis and in antimicrobial immunity. An augmented pathology was observed in mice lacking Toll-like receptor 2 (TLR2) due to an impaired epithelial barrier [75]. Mice lacking the signalling adaptor MYD88, a myeloid differentiation primary response protein 88 , which is essential for signalling by the majority of TLRs [76] [77] had greater bacterial loads both in the colon and in peripheral tissues as the bacteria penetrated deeply into colonic crypts compared to WT mice. Moreover, they suffered from severe colitis and death after infection. The innate immune receptor type-I interleukin-1 receptor (IL-1R), utilizing MyD88 signalling pathway protected mice from severe damage caused by $C$. rodentium [78]. IL-1R deficient mice exhibited increased susceptibility to tissue damage comparable to that of MyD88 knockout mice. Yet, distinct from MyD88 knockout mice, mice deficient in IL-1R did not display amplified pathogen burdens in the colon. In another study, Khan and co-workers exhibited that the bacteria triggered TLR4 and prompted NF- $\kappa B$ nuclear translocation which was dependent on TLR4. Deficiency of TLR4 decreased tissue pathology and inflammatory cell infiltration in gut. Unexpectedly, dissemination of bacteria through colon was hindered in mice lacking TLR4, while the extent of infection was unaffected, suggesting that TLR4-mediated responses were eventually maladaptive to the host [79].

Liu and co-workers demonstrated the biological function of inflammasomes in immune response against $C$. rodentium. Mice deficient in inflammasome components Nlrp3, Nlrc4, and caspase-1 were hyper susceptible to $C$. rodentium induced intestinal inflammation due to impaired production of IL- $1 \beta$ and IL-18 [80]. However, these deficient mice exhibited only mild defects and none of these mice died after infection, indicating that inflammasome is not essential for mice survival after $C$. rodentium infection. In addition, IL- $1 \beta^{-1-}$ and IL- $18^{-/-}$ mice suffered from increased bacterial burdens and had severe histopathology. Therefore, Nlrp3 and Nlrc4 inflammasome-mediated IL- $1 \beta$ and IL-18 response contributed a significant role in host protection against $C$. rodentium [80] [81]. In another study, Kim and co-workers characterized the role of the intracellular Nod-like receptor family members Nod2 in protection against $C$. rodentium infection [82]. Nod2 $2^{-/}$mice displayed diminished intestinal clearance to C. rodentium. The enhanced bacterial load was due to impaired secretion of chemokine ligand 2 (CCL2) from colonic cells and subsequent inflow of monocytes. Fur- 
thermore, IL-12, a cytokine produced by monocytes triggered Th1 immunity vital for bacterial clearance. The adoptive transfer experiments established the significant contribution of Ly6 $\mathrm{C}^{\mathrm{hi}}$ monocytes in the clearance of bacteria in vivo [82]. Table 2 summarizes a comparative analysis of the specific ablation of different innate immune components.

Mice lacking the p50 subunit of the NF- $\mathrm{kB}$ transcription factor, a nuclear factor kappa B, had reduced ability to clear C. rodentium infection [83]. Also a continued bacterial load was reported in mice deficient in p38a, a mitogen-activated protein kinase (MAPK) in intestinal epithelial cells [84]. Interestingly, these animals exhibited no apparent histological lesions, however, failed to recruit $\mathrm{CD} 4^{+} \mathrm{T}$ cells and had impaired chemokines expression. Thus, p38 $\alpha$ in IECs by employing immune cells and adjusting chemokine expression played a part to the host protective immune responses. CXCL9, an ELR (glutamic acid-leucine-arginine) motif chemokine had direct antimicrobial potential against $C$. rodentium and defended crypts from bacterial dissemination. Blockade of this antimicrobial activity by anti-CXCL 9 antibodies escalated host exposure to $C$. rodentium infection

Table 2. Summary of the effects of selective ablation of innate immune components on $C$. rodentium infection in mice.

\begin{tabular}{cc}
\hline Mouse models & Effects of ablation of specific innate immune components on $C$. rodentium infection \\
\hline Mice lacking Toll-like receptor 2 & An augmented pathology due to an impaired epithelial barrier
\end{tabular}
(TLR2)

Mice lacking MYD88

IL-1R deficient mice

Deficiency of TLR4

Mice deficient in Nlrp3, Nlrc4, and caspase-1

IL- $1 \beta^{-/-}$and IL- $18^{-/-}$mice

Nod $2^{-/-}$mice

Mice lacking the p50 subunit of $\mathrm{NF}-\kappa \mathrm{B}$

Mice deficient in p38a

Ablation of specific macrophage/monocyte compartment

Mice lacking PSGL-1 and P, E and L-selectin

Mice lacking $\beta_{7}$ integrin

Mice deficient Muc2
Have greater bacterial loads in colon and peripheral tissues and suffer from severe colitis and death

Increased susceptibility to tissue damage but do not display amplified pathogen burdens in colon.

Decreased tissue pathology and inflammatory cell infiltration in gut. While the extent of infection is [79] unaffected, dissemination of bacteria through colon is hindered

Hyper susceptible to $C$. rodentium induced intestinal inflammation. However, exhibit only mild defects and do not die after infection

Increased bacterial burdens and severe histopathology.

Diminished intestinal clearance to $C$. rodentium. due to impaired secretion of CCL2 from colonic cells

Reduced ability to clear $C$. rodentium infection.

A continued bacterial load with no apparent histological lesions, however, fails to recruit $\mathrm{CD} 4^{+} \mathrm{T}$ cells and impaired chemokines expression.

Neither cell type is essential to trigger immunity

Mice defective in PSGL-1 and P-selectin suffer morbidity, extensive inflammatory responses and augmented bacterial burden, however, mice defective in either E or L-selectin do not exhibit severe infection

Efficiently control infection and clear bacteria 5-6 week after inoculation

Susceptible to the $C$. rodentium-induced colitis and display quick weight loss and exhibit $90 \%$ mortality 
with noticeable bacterial dissemination, augmented bacterial titre, and deteriorated tissue pathology [89]. Surface lymphotoxin expression on group 3 innate lymphoid cells (ILC3s) is critical for early immune responses against C. rodentium [90] [91]. LT aids in IL-22 secretion by intestinal ILCs. Blocking of LT $\beta$ R signaling rapidly diminished intestinal IL-22 production after $C$. rodentium infection [91]. In addition, stimulating LT $\beta$ R signaling induced IL-22 pathway in LT-deficient mice. LT-beta receptor (LTbR) signaling in intestinal epithelial cells was essential for recruitment of neutrophils to the site of infection through secretion of CXCL1 and CXCL2 chemokines. In contrast, surface LT produced by adaptive $\mathrm{B}$ and $\mathrm{T}$ cells was dispensable for protection against gut bacterial infection [90].

The function of macrophages and monocytes during $C$. rodentium infection was investigated using ablation of specific macrophage/monocyte compartment during infection. Although neither cell type was essential to trigger immunity, monocytes and macrophages played a role by secreting IL-12, which prompted Th1 polarization and IFN- $\gamma$ secretion. Thus, monocytes and macrophages contribute in $C$. rodentium immunity by secreting cytokines that direct $\mathrm{T}$ cell polarization [85].

To outline the function of selectins and their ligands during C. rodentium infection, Kum and co-workers investigated infection in mice lacking PSGL-1, a P-selectin glycoprotein ligand-1 and P, E and L-selectin [86]. Mice defective in PSGL-1 and P-selectin suffered morbidity, extensive inflammatory responses and augmented bacterial burden, however, mice defective in either $\mathrm{E}$ or L-selectin did not exhibit severe infection. Also, intestinal inflammation and recruitment of inflammatory cells i.e., neutrophils and macrophages were significantly diminished in P-selectin defective mice which received blocking antibodies to ICAM-1 or LFA-1, suggesting that these adhesion molecules can counterbalance the defect in selectins during leucocyte recruitment [86]. Mice lacking $\beta_{7}$ integrin efficiently controlled infection and cleared bacteria 5-6 week after inoculation [59].

Mice deficient in main intestinal mucin, Muc2, which have an altered intestinal mucus layer, were more susceptible to the $C$. rodentium-induced colitis and displayed quick weight loss and exhibited about $90 \%$ mortality due to a closer interaction of intestinal microbes with the epithelial barrier [87] [88]. $\mathrm{Muc2}^{-/-}$ mice had $10-100$ fold increased C. rodentium load, maximum of which were closely attachded to the mucosa in colon. FITC-Dextran administration exhibited considerably exacerbated disruption in intestinal barrier integrity in $\mathrm{Muc2}^{-/-}$ mice, with explicit bacterial translocation into the colonic mucosa [87] [88].

\subsection{Role of Probiotics and Antibiotic Administration}

Probiotics, a combination of live microorganisms attenuated infection with $C$. rodentium in adult mice and provided a protective role in $C$. rodentium induced death in neonatal mice [92]. In one study, it had been shown that probiotic 
mixture exhibited inhibitory role on the growth of $C$. rodentium. Mice that were administered live probiotics containing a mixture of Lactobacillus rhamnosus and $L$. acidophilus stayed healthy. Pretreatment of mice with probiotics restored colonic integrity and lessened both hyperplasia and inflammatory-cell infiltration in colon [93]. In a recent study, Collins et al. demonstrated that probiotics such as Lactobacillus acidophilus, L. rhamnosus, and Lactobacillus helveticus administered daily in the form of fermented dairy products (FDPs) lessened $C$. rodentium induced colonic hyperplasia and stopped the loss of significant bacterial genera that might lead to disease pathology. However, the FDPs did not result in any noteworthy reduction in $C$. rodentium colonization when estimated by bacterial load [94].

Metronidazole pretreatment augmented exposure to $C$. rodentium-induced colitis compared to that of untreated mice 6 days postinfection and resulted in a diminished number of Porphyromonadaceae and amplified population of lactobacilli [95]. Metronidazole treatment resulted an impaired goblet cell function, decreased Muc2 secretion, a major component of intestinal secretory mucin and thinning of inner mucus layer, resulting in microbially induced immune activation prior to disease induction. Perturbation of the microbiota with metronidazole resulted augmented attachment of bacteria to the intestinal epithelium, resulting in a severe form of C. rodentium-induced colitis in mice [95].

\subsection{Limitations of $C$. rodentium Model}

A limitation to the study of $C$. rodentium infection model is the absence of antigen-specific tools with which to characterize the fate and function of the pathogen/antigen-specific response during infection [96]. The only means that are currently available to address this limitation include transgenic strains of $C$. rodentium that express OVA or GFP [96] [97] [98]. Another probable limitation to study this pathogen could be the loss of antibiotic sensitivity of $C$. rodentium due to the development of worldwide emergence of multi-resistant strains [19]. However, the likelihood of this loss is occasional due to the germ-free condition of the animal houses.

\section{Concluding Remarks}

EPEC and EHEC are the leading cause of diarrhoea in human, affecting children and adults in both developing and developed countries. C. rodentium is an enteric murine pathogen that mimics virulence factors of human EPEC and EHEC and forms comparable attaching and effacing lesions, as a central mechanism of tissue targeting, virulence factors and infection in mice. As a result of this association with other important inflammatory diseases, and that there are cases of more than a million deaths each year from EPEC and EHEC, the knowledge about the pathophysiology of $C$. rodentium infections and following infection how the host immune system responds to it is of immense significance to understand its subsequent function during these inflammatory diseases. 
This review comprehensively covers the salient features of recent discoveries related to $C$. rodentium virulence, epithelial hyperplasia, innate and adaptive immune responses, and the pathophysiology of diarrhoea. It is acknowledged that EPEC and EHEC can be modelled efficiently in mice. Murine $C$. rodentium is a well characterised model of diarrhoeal disease as the molecular, cellular, pathophysiological aspects of the disease have been well studied. Therefore, $C$. rodentium represents an excellent model in which to study the innate and adaptive immune components. We believe that the advances that have been included in this review will give a comprehensive insight to combat the acute diarrhoeal illness in human. Nevertheless, once again $C$. rodentium has been proved to be a useful in vivo model for studying pathogenesis of secretory diarrhoeal diseases/gastrointestinal pathogen and for preventive/mucosal vaccinations and therapeutic approaches.

\section{Conflicts of Interest}

The authors declare no conflict of interest that could be perceived to bias the work.

\section{References}

[1] Nataro, J.P. and Kaper, J.B. (1998) Diarrheagenic Escherichia coli. Clinical Microbiology Reviews, 11, 142-201.

[2] Cassels, F.J. and Wolf, M.K. (1995) Colonization Factors of Diarrheagenic E. coli and Their Intestinal Receptors. Journal of Industrial Microbiology, 15, 214-226. https://doi.org/10.1007/BF01569828

[3] Frankel, G., et al. (1998) Enteropathogenic and Enterohaemorrhagic Escherichia coli: More Subversive Elements. Molecular Microbiology, 30, 911-921. https://doi.org/10.1046/j.1365-2958.1998.01144.x

[4] Clarke, S.C., et al. (2003) Virulence of Enteropathogenic Escherichia coli, a Global Pathogen. Clinical Microbiology Reviews, 16, 365-378. https://doi.org/10.1128/CMR.16.3.365-378.2003

[5] Chen, H.D. and Frankel, G. (2005) Enteropathogenic Escherichia coli: Unravelling Pathogenesis. FEMS Microbiology Reviews, 29, 83-98.

https://doi.org/10.1016/j.femsre.2004.07.002

[6] Mead, P.S., et al. (1999) Food-Related Illness and Death in the United States Reply to Dr. Hedberg. Emerging Infectious Diseases, 5, 841-842.

https://doi.org/10.3201/eid0506.990625

[7] Rangel, J.M., et al. (2005) Epidemiology of Escherichia coli O157:H7 Outbreaks, United States, 1982-2002. Emerging Infectious Diseases, 11, 603-609. https://doi.org/10.3201/eid1104.040739

[8] Welinder-Olsson, C. and Kaijser, B. (2005) Enterohemorrhagic Escherichia coli (EHEC). Scandinavian Journal of Infectious Diseases, 37, 405-416. https://doi.org/10.1080/00365540510038523

[9] Schmidt, H., et al. (1996) Pore-Forming Properties of the Plasmid-Encoded Hemolysin of Enterohemorrhagic Escherichia coli O157:H7. European Journal of Biochemistry, 241, 594-601. https://doi.org/10.1111/j.1432-1033.1996.00594.x

[10] Kaper, J.B., Nataro, J.P. and Mobley, H.L. (2004) Pathogenic Escherichia coli. Na- 
ture Reviews Microbiology, 2, 123-140. https://doi.org/10.1038/nrmicro818

[11] Mundy, R., et al. (2006) Comparison of Colonization Dynamics and Pathology of Mice Infected with Enteropathogenic Escherichia coli, Enterohaemorrhagic E. coli and Citrobacter rodentium. FEMS Microbiology Letters, 265, 126-132. https://doi.org/10.1111/j.1574-6968.2006.00481.x

[12] Mundy, R., et al. (2005) Citrobacter rodentium of Mice and Man. Cellular Microbiology, 7, 1697-1706. https://doi.org/10.1111/j.1462-5822.2005.00625.x

[13] Luperchio, S.A. and Schauer, D.B. (2001) Molecular Pathogenesis of Citrobacter rodentium and Transmissible Murine Colonic Hyperplasia. Microbes and Infection, 3, 333-340. https://doi.org/10.1016/S1286-4579(01)01387-9

[14] Savkovic, S.D., et al. (2005) Mouse Model of Enteropathogenic Escherichia coli Infection. Infection and Immunity, 73, 1161-1170. https://doi.org/10.1128/IAI.73.2.1161-1170.2005

[15] Schauer, D.B. and Falkow, S. (1993) Attaching and Effacing Locus of a Citrobacter freundii Biotype that Causes Transmissible Murine Colonic Hyperplasia. Infection and Immunity, 61, 2486-2492.

[16] Barthold, S.W., Coleman, G.L., Jacoby, R.O., Livestone, E.M. and Jonas, A.M. (1978) Transmissible Murine Colonic Hyperplasia. Veterinary Pathology, 15, 223-236. https://doi.org/10.1177/030098587801500209

[17] Wiles, S., et al. (2004) Organ Specificity, Colonization and Clearance Dynamics in Vivo Following Oral Challenges with the Murine Pathogen Citrobacter rodentium. Cellular Microbiology, 6, 963-972. https://doi.org/10.1111/j.1462-5822.2004.00414.x

[18] Wiles, S., Pickard, K.M., Peng, K., MacDonald, T.T. and Frankel, G. (2006) In Vivo Bioluminescence Imaging of the Murine Pathogen Citrobacter rodentium. Infection and Immunity, 74, 5391-5396. https://doi.org/10.1128/IAI.00848-06

[19] Buschor, S., et al. (2017) Innate Immunity Restricts Citrobacter rodentium A/E Pathogenesis Initiation to an Early Window of Opportunity. PLOS Pathogens, 13, e1006476. https://doi.org/10.1371/journal.ppat.1006476

[20] Zahavi, E.E., et al. (2011) Bundle-Forming Pilus Retraction Enhances Enteropathogenic Escherichia coli Infectivity. Molecular Biology of the Cell, 22, 2436-2447. https://doi.org/10.1091/mbc.e11-01-0001

[21] Vallance, B.A. and Finlay, B.B. (2000) Exploitation of Host Cells by Enteropathogenic Escherichia coli. Proceedings of the National Academy of Sciences, 97, 8799-8806. https://doi.org/10.1073/pnas.97.16.8799

[22] Garmendia, J., Frankel, G. and Crepin, V.F. (2005) Enteropathogenic and Enterohemorrhagic Escherichia coli Infections: Translocation, Translocation, Translocation. Infection and Immunity, 73, 2573-2585. https://doi.org/10.1128/IAI.73.5.2573-2585.2005

[23] Vallance, B.A., Deng, W., Jacobson, K. and Finlay, B.B. (2003) Host Susceptibility to the Attaching and Effacing Bacterial Pathogen Citrobacter rodentium. Infection and Immunity, 71, 3443-3453. https://doi.org/10.1128/IAI.71.6.3443-3453.2003

[24] Franzin, F.M. and Sircili, M.P. (2015) Locus of Enterocyte Effacement: A Pathogenicity Island Involved in the Virulence of Enteropathogenic and Enterohemorragic Escherichia coli Subjected to a Complex Network of Gene Regulation. BioMed Research International, 2015, Article ID: 534738. https://doi.org/10.1155/2015/534738

[25] Deng, W., Li, Y., Vallance, B.A. and Finlay, B.B. (2001) Locus of Enterocyte Effacement from Citrobacter rodentium: Sequence Analysis and Evidence for Horizontal 
Transfer among Attaching and Effacing Pathogens. Infection and Immunity, 69, 6323-6335. https://doi.org/10.1128/IAI.69.10.6323-6335.2001

[26] Deng, W., et al. (2004) Dissecting Virulence: Systematic and Functional Analyses of a Pathogenicity Island. Proceedings of the National Academy of Sciences, 101, 3597-3602. https://doi.org/10.1073/pnas.0400326101

[27] Elliott, S.J., et al. (2000) The Locus of Enterocyte Effacement (LEE)-Encoded Regulator Controls Expression of Both LEE- and Non-LEE-Encoded Virulence Factors in Enteropathogenic and Enterohemorrhagic Escherichia coli. Infection and Immunity, 68, 6115-6126. https://doi.org/10.1128/IAI.68.11.6115-6126.2000

[28] Gaytan, M.O., Martínez-Santos, V.I., Soto, E. and González-Pedrajo, B. (2016) Type Three Secretion System in Attaching and Effacing Pathogens. Frontiers in Cellular and Infection Microbiology, 6, 129. https://doi.org/10.3389/fcimb.2016.00129

[29] Shames, S.R., Croxen, M.A., Deng, W. and Finlay, B.B. (2011) The Type III System-Secreted Effector EspZ Localizes to Host Mitochondria and Interacts with the Translocase of Inner Mitochondrial Membrane 17b. Infection and Immunity, 79, 4784-4790. https://doi.org/10.1128/IAI.05761-11

[30] MacDonald, T.T., Frankel, G., Dougan, G., Goncalves, N.S. and Simmons, C. (2003) Host Defences to Citrobacter rodentium. International Journal of Medical Microbiology, 293, 87-93. https://doi.org/10.1078/1438-4221-00247

[31] Oswald, E., Schmidt, H., Morabito, S., Karch, H., Marchès, O. and Caprioli, A. (2000) Typing of Intimin Genes in Human and Animal Enterohemorrhagic and Enteropathogenic Escherichia coli: Characterization of a New Intimin Variant. Infection and Immunity, 68, 64-71. https://doi.org/10.1128/IAI.68.1.64-71.2000

[32] Yi, Y., et al. (2010) Crystal Structure of EHEC Intimin: Insights into the Complementarity between EPEC and EHEC. PLOS ONE, 5, e15285.

https://doi.org/10.1371/journal.pone.0015285

[33] Adu-Bobie, J., et al. (1998) Detection of Intimins Alpha, Beta, Gamma, and Delta, four Intimin Derivatives Expressed by Attaching and Effacing Microbial Pathogens. Journal of Clinical Microbiology, 36, 662-668.

[34] Kenny, B. and Finlay, B.B. (1997) Intimin-Dependent Binding of Enteropathogenic Escherichia coli to Host Cells Triggers Novel Signaling Events, Including Tyrosine Phosphorylation of Phospholipase C-Gamma1. Infection and Immunity, 65, 2528-2536.

[35] Kenny, B., DeVinney, R., Stein, M., Reinscheid, D.J., Frey, E.A. and Finlay, B.B. (1997) Enteropathogenic E. coli (EPEC) Transfers Its Receptor for Intimate Adherence into Mammalian Cells. Cell, 91, 511-520. https://doi.org/10.1016/S0092-8674(00)80437-7

[36] Campellone, K.G., Giese, A., Tipper, D.J. and Leong, J.M. (2002) A Tyrosine-Phosphorylated 12-Amino-Acid Sequence of Enteropathogenic Escherichia coli Tir Binds the Host Adaptor Protein Nck and Is Required for Nck Localization to Actin Pedestals. Molecular Microbiology, 43, 1227-1241. https://doi.org/10.1046/j.1365-2958.2002.02817.x

[37] Martinez-Quiles, N., Feuerbacher, L.A., Benito-León, M. and Hardwidge, P.R. (2014) Contribution of Crk Adaptor Proteins to Host Cell and Bacteria Interactions. BioMed Research International, 2014, Article ID: 372901. https://doi.org/10.1155/2014/372901

[38] Buday, L., Wunderlich, L. and Tamas, P. (2002) The Nck Family of Adapter Proteins: Regulators of Actin Cytoskeleton. Cell Signal, 14, 723-731. 
https://doi.org/10.1016/S0898-6568(02)00027-X

[39] Gruenheid, S., et al. (2001) Enteropathogenic E. coli Tir Binds Nck to Initiate Actin Pedestal Formation in Host Cells. Nature Cell Biology, 3, 856-859. https://doi.org/10.1038/ncb0901-856

[40] Nieto-Pelegrin, E., Kenny, B. and Martinez-Quiles, N. (2014) Nck Adaptors, Besides Promoting N-WASP Mediated Actin-Nucleation Activity at Pedestals, Influence the Cellular Levels of Enteropathogenic Escherichia coli Tir Effector. Cell Adhesion \& Migration, 8, 404-417. https://doi.org/10.4161/19336918.2014.969993

[41] Collins, J.W., et al. (2014) Citrobacter rodentium: Infection, Inflammation and the Microbiota. Nature Reviews Microbiology, 12, 612-623.

https://doi.org/10.1038/nrmicro3315

[42] Donnenberg, M.S. and Whittam, T.S. (2001) Pathogenesis and Evolution of Virulence in Enteropathogenic and Enterohemorrhagic Escherichia coli. Journal of Clinical Investigation, 107, 539-548. https://doi.org/10.1172/JCI12404

[43] Ugalde-Silva, P., Gonzalez-Lugo, O. and Navarro-Garcia, F. (2016) Tight Junction Disruption Induced by Type 3 Secretion System Effectors Injected by Enteropathogenic and Enterohemorrhagic Escherichia coli. Frontiers in Cellular and Infection Microbiology, 6, 87. https://doi.org/10.3389/fcimb.2016.00087

[44] Abe, H., et al. (2008) Global Regulation by Horizontally Transferred Regulators Establishes the Pathogenicity of Escherichia coli. DNA Research, 15, 25-38.

https://doi.org/10.1093/dnares/dsm033

[45] Torres, A.G., et al. (2007) Ler and H-NS, Regulators Controlling Expression of the Long Polar Fimbriae of Escherichia coli O157:H7. Journal of Bacteriology, 189, 5916-5928. https://doi.org/10.1128/JB.00245-07

[46] Holmes, A., Lindestam Arlehamn, C.S., Wang, D., Mitchell, T.J., Evans, T.J. and Roe, A.J. (2012) Expression and Regulation of the Escherichia coli O157:H7 Effector Proteins NleH1 and NleH2. PLoS ONE, 7, e33408.

https://doi.org/10.1371/journal.pone.0033408

[47] Yang, J., Tauschek, M., Hart, E., Hartland, E.L. and Robins-Browne, R.M. (2010) Virulence Regulation in Citrobacter rodentium: The Art of Timing. Microbial Biotechnology, 3, 259-268. https://doi.org/10.1111/j.1751-7915.2009.00114.x

[48] Gruenheid, S., et al. (2004) Identification and Characterization of NleA, a Non-LEE-Encoded Type III Translocated Virulence Factor of Enterohaemorrhagic Escherichia coli O157:H7. Molecular Microbiology, 51, 1233-1249. https://doi.org/10.1046/j.1365-2958.2003.03911.x

[49] Kelly, M., et al. (2006) Essential Role of the Type III Secretion System Effector NleB in Colonization of Mice by Citrobacter rodentium. Infection and Immunity, 74, 2328-2337. https://doi.org/10.1128/IAI.74.4.2328-2337.2006

[50] Thanabalasuriar, A., et al. (2012) Sec24 Interaction Is Essential for Localization and Virulence-Associated Function of the Bacterial Effector Protein NleA. Cellular Microbiology, 14, 1206-1218. https://doi.org/10.1111/j.1462-5822.2012.01789.x

[51] Lee, S.F., et al. (2008) A C-Terminal Class I PDZ Binding Motif of EspI/NleA Modulates the Virulence of Attaching and Effacing Escherichia coli and Citrobacter rodentium. Cellular Microbiology, 10, 499-513.

[52] Newman, J.V., Zabel, B.A., Jha, S.S. and Schauer, D.B. (1999) Citrobacter rodentium espB Is Necessary for Signal Transduction and for Infection of Laboratory Mice. Infection and Immunity, 67, 6019-6025.

[53] Dahan, S., et al. (2005) EspJ Is a Prophage-Carried Type III Effector Protein of At- 
taching and Effacing Pathogens That Modulates Infection Dynamics. Infection and Immunity, 73, 679-686. https://doi.org/10.1128/IAI.73.2.679-686.2005

[54] Shaw, R.K., et al. (2005) Enteropathogenic Escherichia coli Type III Effectors EspG and EspG2 Disrupt the Microtubule Network of Intestinal Epithelial Cells. Infection and Immunity, 73, 4385-4390. https://doi.org/10.1128/IAI.73.7.4385-4390.2005

[55] Newton, H.J., et al. (2010) The Type III Effectors NleE and NleB from Enteropathogenic E. coli and OspZ from Shigella Block Nuclear Translocation of NF-kappaB p65. PLOS Pathogens, 6, e1000898. https://doi.org/10.1371/journal.ppat.1000898

[56] Yen, H., Ooka, T., Iguchi, A., Hayashi, T., Sugimoto, N. and Tobe, T. (2010) NleC, a Type III Secretion Protease, Compromises NF-kappaB Activation by Targeting p65/RelA. PLOS Pathogens, 6, e1001231. https://doi.org/10.1371/journal.ppat.1001231

[57] Pearson, J.S., et al. (2013) A Type III Effector Antagonizes Death Receptor Signalling during Bacterial Gut Infection. Nature, 501, 247-251. https://doi.org/10.1038/nature12524

[58] Symonds, E.L., Riedel, C.U., O’Mahony, D., Lapthorne, S., O’Mahony, L. and Shanahan, F. (2009) Involvement of T Helper Type 17 and Regulatory T Cell Activity in Citrobacter rodentium Invasion and Inflammatory Damage. Clinical \& EXperimental Immunology, 157, 148-154. https://doi.org/10.1111/j.1365-2249.2009.03934.x

[59] Bry, L. and Brenner, M.B. (2004) Critical Role of T Cell-Dependent Serum Antibody, But Not the Gut-Associated Lymphoid Tissue, for Surviving Acute Mucosal Infection with Citrobacter rodentium, an Attaching and Effacing Pathogen. The Journal of Immunology, 172, 433-441. https://doi.org/10.4049/jimmunol.172.1.433

[60] Shen-Tu, G., Schauer, D.B., Jones, N.L. and Sherman, P.M. (2010) Detergent-Resistant Microdomains Mediate Activation of Host Cell Signaling in Response to Attaching-Effacing Bacteria. Laboratory Investigation, 90, 266-281. https://doi.org/10.1038/labinvest.2009.131

[61] Umar, S., Scott, J., Sellin, J.H., Dubinsky, W.P. and Morris, A.P. (2000) Murine Colonic Mucosa Hyperproliferation. I. Elevated CFTR Expression and Enhanced cAMP-Dependent Cl(-) Secretion. American Journal of Physiology-Gastrointestinal and Liver Physiology, 278, G753-G764. https://doi.org/10.1152/ajpgi.2000.278.5.G753

[62] Goosney, D.L., Gruenheid, S. and Finlay, B.B. (2000) Gut Feelings: Enteropathogenic E. coli (EPEC) Interactions with the Host. Annual Review of Cell and Developmental Biology, 16, 173-189. https://doi.org/10.1146/annurev.cellbio.16.1.173

[63] Simmons, C.P., et al. (2003) Central Role for B Lymphocytes and CD4+ T Cells in Immunity to Infection by the Attaching and Effacing Pathogen Citrobacter rodentium. Infection and Immunity, 71, 5077-5086. https://doi.org/10.1128/IAI.71.9.5077-5086.2003

[64] Higgins, L.M., Frankel, G., Douce, G., Dougan, G. and MacDonald, T.T. (1999) Citrobacter rodentium Infection in Mice Elicits a Mucosal Th1 Cytokine Response and Lesions Similar to Those in Murine Inflammatory Bowel Disease. Infection and Immunity, 67, 3031-3039.

[65] Maaser, C., et al. (2004) Clearance of Citrobacter rodentium Requires B Cells But Not Secretory Immunoglobulin A (IgA) or IgM Antibodies. Infection and Immunity, 72, 3315-3324. https://doi.org/10.1128/IAI.72.6.3315-3324.2004

[66] Smith, A.D., Botero, S., Shea-Donohue, T. and Urban, J.F. (2011) The Pathogenicity 
of an Enteric Citrobacter rodentium Infection Is Enhanced by Deficiencies in the Antioxidants Selenium and Vitamin E. Infection and Immunity, 79, 1471-1478. https://doi.org/10.1128/IAI.01017-10

[67] Vallance, B.A., Deng, W., Knodler, L.A. and Finlay, B.B. (2002) Mice Lacking T and B Lymphocytes Develop Transient Colitis and Crypt Hyperplasia Yet Suffer Impaired Bacterial Clearance during Citrobacter rodentium Infection. Infection and Immunity, 70, 2070-2081. https://doi.org/10.1128/IAI.70.4.2070-2081.2002

[68] Simmons, C.P., et al. (2002) Impaired Resistance and Enhanced Pathology during Infection with a Noninvasive, Attaching-Effacing Enteric Bacterial Pathogen, Citrobacter rodentium, in Mice Lacking IL-12 or IFN-Gamma. The Journal of Immunology, 168, 1804-1812. https://doi.org/10.4049/jimmunol.168.4.1804

[69] Zheng, Y., et al. (2008) Interleukin-22 Mediates Early Host Defense against Attaching and Effacing Bacterial Pathogens. Nature Medicine, 14, 282-289. https://doi.org/10.1038/nm1720

[70] Li, L., et al. (2014) Cytokine IL-6 Is Required in Citrobacter rodentium Infection-Induced Intestinal Th17 Responses and Promotes IL-22 Expression in Inflammatory Bowel Disease. Molecular Medicine Reports, 9, 831-836.

https://doi.org/10.3892/mmr.2014.1898

[71] Wang, Z., et al. (2014) Regulatory T Cells Promote a Protective Th17-Associated Immune Response to Intestinal Bacterial Infection with C. rodentium. Mucosal Immunology, 7, 1290-1301. https://doi.org/10.1038/mi.2014.17

[72] Dann, S.M., et al. (2014) Attenuation of Intestinal Inflammation in Interleukin-10-Deficient Mice Infected with Citrobacter rodentium. Infection and Immunity, 82, 1949-1958. https://doi.org/10.1128/IAI.00066-14

[73] Basu, R., et al. (2012) Th22 Cells Are an Important Source of IL-22 for Host Protection against Enteropathogenic Bacteria. Immunity, 37, 1061-1075. https://doi.org/10.1016/j.immuni.2012.08.024

[74] Ishigame, H., et al. (2009) Differential Roles of Interleukin-17A and -17F in Host Defense against Mucoepithelial Bacterial Infection and Allergic Responses. Immunity, 30, 108-119. https://doi.org/10.1016/j.immuni.2008.11.009

[75] Gibson, D.L., et al. (2008) Toll-Like Receptor 2 Plays a Critical Role in Maintaining Mucosal Integrity during Citrobacter rodentium-Induced Colitis. Cellular Microbiology, 10, 388-403.

[76] Gibson, D.L., Ma, C., Bergstrom, K.S., Huang, J.T., Man, C. and Vallance, B.A. (2008) MyD88 Signalling Plays a Critical Role in Host Defence by Controlling Pathogen Burden and Promoting Epithelial Cell Homeostasis during Citrobacter rodentium-Induced Colitis. Cellular Microbiology, 10, 618-631. https://doi.org/10.1111/j.1462-5822.2007.01071.x

[77] Lebeis, S.L., Bommarius, B., Parkos, C.A., Sherman, M.A. and Kalman, D. (2007) TLR Signaling Mediated by MyD88 Is Required for a Protective Innate Immune Response by Neutrophils to Citrobacter rodentium. The Journal of Immunology, 179, 566-577. https://doi.org/10.4049/jimmunol.179.1.566

[78] Lebeis, S.L., Powell, K.R., Merlin, D., Sherman, M.A. and Kalman, D. (2009) Interleukin-1 Receptor Signaling Protects Mice from Lethal Intestinal Damage Caused by the Attaching and Effacing Pathogen Citrobacter rodentium. Infection and Immunity, 77, 604-614. https://doi.org/10.1128/IAI.00907-08

[79] Khan, M.A., et al. (2006) Toll-Like Receptor 4 Contributes to Colitis Development But Not to Host Defense during Citrobacter rodentium Infection in Mice. Infection 
and Immunity, 74, 2522-2536. https://doi.org/10.1128/IAI.74.5.2522-2536.2006

[80] Liu, Z., et al. (2012) Role of Inflammasomes in Host Defense against Citrobacter rodentium Infection. The Journal of Biological Chemistry, 287, 16955-16964. https://doi.org/10.1074/jbc.M112.358705

[81] Nordlander, S., Pott, J. and Maloy, K.J. (2014) NLRC4 Expression in Intestinal Epithelial Cells Mediates Protection against an Enteric Pathogen. Mucosal Immunology, 7, 775-785. https://doi.org/10.1038/mi.2013.95

[82] Kim, Y.G., et al. (2011) The Nod2 Sensor Promotes Intestinal Pathogen Eradication via the Chemokine CCL2-Dependent Recruitment of Inflammatory Monocytes. Immunity, 34, 769-780. https://doi.org/10.1016/j.immuni.2011.04.013

[83] Dennis, A., et al. (2008) The p50 Subunit of NF-kappaB Is Critical for in Vivo Clearance of the Noninvasive Enteric Pathogen Citrobacter rodentium. Infection and Immunity, 76, 4978-4988. https://doi.org/10.1128/IAI.00736-08

[84] Kang, Y.J., et al. (2010) Epithelial p38 Alpha Controls Immune Cell Recruitment in the Colonic Mucosa. PLOS Pathogens, 6, e1000934.

https://doi.org/10.1371/journal.ppat.1000934

[85] Schreiber, H.A., et al. (2013) Intestinal Monocytes and Macrophages Are Required for T Cell Polarization in Response to Citrobacter rodentium. The Journal of $E_{X-}$ perimental Medicine, 210, 2025-2039. https://doi.org/10.1084/jem.20130903

[86] Kum, W.W., Lo, B.C., Deng, W., Ziltener, H.J. and Finlay, B.B. (2010) Impaired Innate Immune Response and Enhanced Pathology during Citrobacter rodentium Infection in Mice Lacking Functional P-Selectin. Cellular Microbiology, 12, 1250-1271. https://doi.org/10.1111/j.1462-5822.2010.01466.x

[87] Bergstrom, K.S., et al. (2010) Muc2 Protects against Lethal Infectious Colitis by Disassociating Pathogenic and Commensal Bacteria from the Colonic Mucosa. PLOS Pathogens, 6, e1000902. https://doi.org/10.1371/journal.ppat.1000902

[88] Van der Sluis, M., et al. (2006) Muc2-Deficient Mice Spontaneously Develop Colitis, Indicating That MUC2 Is Critical for Colonic Protection. Gastroenterology, 131, 117-129. https://doi.org/10.1053/j.gastro.2006.04.020

[89] Reid-Yu, S.A., Tuinema, B.R., Small, C.N., Xing, L. and Coombes, B.K. (2015) CXCL9 Contributes to Antimicrobial Protection of the Gut during Citrobacter rodentium Infection Independent of Chemokine-Receptor Signaling. PLOS Pathogens, 11, e1004648. https://doi.org/10.1371/journal.ppat.1004648

[90] Wang, Y., et al. (2010) Lymphotoxin Beta Receptor Signaling in Intestinal Epithelial Cells Orchestrates Innate Immune Responses against Mucosal Bacterial Infection. Immunity, 32, 403-413. https://doi.org/10.1016/j.immuni.2010.02.011

[91] Tumanov, A.V., et al. (2011) Lymphotoxin Controls the IL-22 Protection Pathway in Gut Innate Lymphoid Cells during Mucosal Pathogen Challenge. Cell Host \& Microbe, 10, 44-53. https://doi.org/10.1016/j.chom.2011.06.002

[92] Gareau, M.G., Wine, E., Reardon, C. and Sherman, P.M. (2010) Probiotics Prevent Death Caused by Citrobacter rodentium Infection in Neonatal Mice. The Journal of Infectious Diseases, 201, 81-91. https://doi.org/10.1086/648614

[93] Johnson-Henry, K.C., et al. (2005) Amelioration of the Effects of Citrobacter rodentium Infection in Mice by Pretreatment with Probiotics. The Journal of Infectious Diseases, 191, 2106-2117. https://doi.org/10.1086/430318

[94] Collins, J.W., et al. (2014) Fermented Dairy Products Modulate Citrobacter rodentium-Induced Colonic Hyperplasia. The Journal of Infectious Diseases, 210, 1029-1041. 
https://doi.org/10.1093/infdis/jiu205

[95] Wlodarska, M., et al. (2011) Antibiotic Treatment Alters the Colonic Mucus Layer and Predisposes the Host to Exacerbated Citrobacter rodentium-Induced Colitis. Infection and Immunity, 79, 1536-1545. https://doi.org/10.1128/IAI.01104-10

[96] Silberger, D.J., Zindl, C.L. and Weaver, C.T. (2017) Citrobacter rodentium: A Model Enteropathogen for Understanding the Interplay of Innate and Adaptive Components of Type 3 Immunity. Mucosal Immunology, 10, 1108-1117. https://doi.org/10.1038/mi.2017.47

[97] Shiomi, H., et al. (2010) Gamma Interferon Produced by Antigen-Specific CD4+ T Cells Regulates the Mucosal Immune Responses to Citrobacter rodentium Infection. Infection and Immunity, 78, 2653-2666. https://doi.org/10.1128/IAI.01343-09

[98] Yang, Y., et al. (2014) Focused Specificity of Intestinal TH17 Cells towards Commensal Bacterial Antigens. Nature, 510, 152-156.

https://doi.org/10.1038/nature13279 